\title{
Near-infrared light spectroscopy and stimulation in cognitive neuroscience-the need for an integrative view?
}

\section{Introduction}

Brain research based on non-invasive light-emitting probes has seen its start in the Seventies [1] and since then this technology has been used in several experiments and applications. This commentary will refer to the different uses of near-infrared light technology, either NIRS or photobiomodulation (PBM), hinting at a possible functional overlap of the two.

Due to its versatility and compactness, near-infrared spectroscopy (NIRS) has been used to explore the effects of physical exercise before, during and/or after the actual activity.
Here we will revise a series of studies about the effects of physical activity on the brain and behavioral outcomes, arguing that there might be a possible extra effect operated by NIRS itself, which has been uncontrolled.

\section{NIRS and cerebral pulsatility}

Using a sample of older adults with cardiovascular risk factors, Mohammadi and colleagues [2] have recently measured the impact of 12 months of a short, aerobic systematic physical activity on cerebral pulsatility and examined its relationship with cognition. Cerebral pulsatility, also known as cerebral pulse amplitude, is related to the enlargement of the arteries following the pulse pressure wave [3]. The authors found that one year of exercise is linked to a reduction in cerebral pulsatility, and that such reduction is related to a better executive functioning. In particular, such exercise-driven reduction of cerebral pulsatility, is associated with shorter response times at the Stroop test. They also found that the cerebral pulsatility was lower after a short walk, but this decrease was more pronounced after one year of regular exercise [2]. By extracting cerebral pulsatile waveforms it is possible to obtain indices of several parameters related to cerebrovascular health, namely, arterial compliance, pulse amplitude and pulse transit time [4]. The clinical relevance of Mohammadi's work resides in the fact that pulse amplitude correlates positively with age [5] and that a consistently higher pulsatility in the cerebral blood flow (CBF) could be associated with microvascular damage and cognitive decline [6]. But apart from their novel and potentially clinically-relevant findings, part of the originality of this work consists in the use that the authors make of NIRS technology to gauge cerebral pulsatility which, until now, has been traditionally provided by Transcranial Doppler (TCD) $[7,8]$. The measurement of such an index via optical imaging is not completely novel $[4,5]$, but, to our knowledge, this is the first study to do it with physical activity and cognition. 


\section{NIRS: the 'conventional' use}

Originally intended to gauge clinically-relevant tissue oxygenation measures, NIRS has evolved into its 'functional' use with neuroimaging studies [9]. In cognitive neuroscience, fNIRS is used to gather information about the levels of oxygenation of cortical areas. fNIRS is based on the principle that most biological tissues are semi-transparent to infrared light, whose wavelengths range between 600 and $1000 \mathrm{~nm}$ $[10,11]$. Oxygenated and deoxygenated hemoglobin (oxyand deoxy- $\mathrm{Hb}$, or $\mathrm{HbO}$ and $\mathrm{HbR}$ ) in the blood vessels act as chromophores (light absorbers) and the absorption variations and extinction coefficients are transformed in concentration of $\mathrm{HbO}$ and HbR using the Beer-Lamber law [12]. Thus, when photons are projected into the head, they are either scattered by the different layers of the head or absorbed mainly by oxy- and deoxy-Hb [13]. By measuring the quantity of returning photons as a function of wavelength, the average regional hemoglobin oxygen saturation can be estimated by the characterization of the spectral absorption of the underlying tissue [14].

Even though motion and mechanical artifacts still represent a problem and need to be removed through ad-hoc filters [15-17], fNIRS is less prone to motion artifacts than other neuroimaging techniques. It can be utilized during physical activity [18]. Also, since both aerobic and anaerobic exercise have been proven to protect and enhance cognitive and brain functions [19-21]. A few studies have lately investigated the effects of short bouts of regular physical activity on cerebral hemodynamic and cognition. Because infrared light is strongly absorbed by hair and hair follicles [22], NIRS probes are usually placed on the forehead of the participants. While motor and sensory-motor tasks may require the application of the device on frontal and temporoparietal cortices $[23,24]$, most of the studies using fNIRS focus its investigation on the metabolism (here meant as oxygen consumption) and hemoglobin of the prefrontal cortex (PFC) $[25,26]$. According to the principle of "neurovascular coupling" changes in neural activity are associated with an alteration in the cerebral blood flow so that a high level of activity in a specific brain region will determine a local increase of blood flow in that region [27]. In other words, a brain area that is 'more active' will receive more blood supply. Since the PFC is thought to be the neural substrate of executive functions (EF) $[28,29]$, it is no surprise that most of the articles published in cognitive neuroscience using fNIRS report data relative to EF, investigating the possible correlations between the behavioral performance and the activation of the PFC.

\section{4. fNIRS and physical activity}

The first to apply fNIRS measurements to see the effects of a single bout of exercise on cognition has been the one carried out by Yanagisawa and colleagues [30], who found a significant improvement in cognitive performance after a single session of physical activity and an enhanced exercise-driven activation in the left dorsolateral prefrontal cortex (DLPFC).
Importantly, the greater activation matched the improved cognitive performance, suggesting a direct relationship between the exercise-driven activation of the DLPFC and the improved Stroop performance [30]. Further evidence in favor of the positive effect of physical activity on cognition and brain activity was provided by other NIRS studies, where it was found that: NIRS-derived prefrontal cortical hemodynamics are influenced by exercise intensity, age of participants and type of cognitive task [31]; activity in PFC is progressively enhanced during increasing levels of exercise (cycling) [32]; high-fit older women show significant increases in the cortical right PFC activations as a function of task difficulty compared to low-fit group [33]; the right inferior frontal gyrus is activated during neuropsychological testing only in high fit women [34]; a continuous rather than an intermittent type of exercise can lead to a higher cortical oxygenation level and a better performance at the Stroop tasks compared to pre-exercise indices [35]. On the other hand, PFC oxygenation and EF-related performance decline when physical activity becomes too intense [36-38]. In some cases, even moderate exercise could negatively affect EF and DLPFC activations [39].

The effects of physical activity are just one of the possible research strands to find valid interventions to improve cognitive functioning, and fNIRS is a useful tool to provide useful insights into the neural underpinnings of such improvements. However, as we will discuss in the next part of this manuscript, many of the findings reported above could have missed an important mechanism that can take place when projecting NIR light into biological tissue. Therefore, we argue that many of the results obtained through fNIRS may have overlooked a possible active contribution of fNIRS itself.

Other potential challenges and limitations are also present when fNIRS is involved, but these have been raised and discussed elsewhere [40, 41].

\section{Near-infrared light photobiomodulation}

As outlined in the previous paragraphs, fNIRS has been proposed in the cognitive neuroscience field as a reliable tool relying on continuous-wave (yet frequency-domain and time-domain systems are also available, see [42]) monochromatic near-infrared light, to have live monitoring of the changes in the concentration of oxyHb and deoxyHb. However, when applied to the head, light in the infrared spectrum can also determine changes in neural activity. A proof of this comes from studies on photobiomodulation (PBM) [43]. PBM is defined as the administration of visible red or Near-infrared (NIR) light to stimulate a biological system [44]. Even though light absorption can yield tissue heating [45], PBM is a clinically safe technique as it relies on specific wavelengths which normally do not produce heating [46]. Indeed, studies on transcranial near-infrared light therapy (NILT) found no negative effects on the structure or function of the brain when such light stimulation was deliv- 
ered [47-50]. The primary biological target of PBM is the cytochrome c oxidase (CCO), an enzyme found in the inner membrane of mitochondria that is essential for cellular metabolism [51, 52]. CCO, the last unit of the electron transport chain, has a role in completing ATP synthesis in the mitochondria by swapping electrons from cytochrome $\mathrm{c}$ to molecular oxygen. So, by applying PBM through NIR light, it is possible to stimulate $\mathrm{CCO}$, leading to oxygen consumption and increased energy production via mitochondrial oxidative phosphorylation [53].

Moreover, since CCO is an adaptive enzyme, the effects are long-lasting. It is also worth noting that the indirect effects of $\mathrm{PBM}$ include those on important messengers such as calcium, reactive oxygen species and cyclic adenosine monophosphate $[54,55]$. All of them are relevant to diverse biological cascades, which are involved in maintaining homeostasis and activating gene factors and systematic responses, such as $\mathrm{CBF}$. Indeed, $\mathrm{CBF}$ has been proved to be deficient in neurocognitive disorders [44, 55-58] and certain disorders such as adults with cardiovascular risk factors [59], a type of population considered in Mohammadi et al.'s article. If the type of design would exclude a role of PBM (via NIRS) in Mohammadi and colleagues' work, other studies have supported the role of PBM through NIR light stimulation in improving the CBF. For example, Tian and coworkers [60] reported a significant reduction in deoxygenation and increase in hemoglobin oxygenation in both cerebral hemispheres during irradiation (10 minutes) and post-irradiation (6 minutes) after applying $1064 \mathrm{~nm}$ laser for 10 minutes to the forehead.

Further experiments have proved that PBM delivered by NIR-light-emitting devices could elicit changes in brain activity. Indeed, Zomorrodi and colleagues [61] performed a controlled-placebo study, where the brain response was measured following a NIR light pulse $(810 \mathrm{~nm}$ wavelength, $40 \mathrm{~Hz}$ ) applied on different regions (frontal, left/right temporal, precuneus cortex, and intranasal) for a 20-minute session. PBM led to frequency-dependent effects on endogenous brain activity. In an Alzheimer's disease patient [62], low frequencies power reduction (i.e., delta and theta) and a higher frequencies power increase (i.e., alpha, beta, and gamma) were recorded following light stimulation. These findings might suggest that PBM could also modulate cortical oscillations and then brain connectivity in a frequency-dependent manner. So, it is possible to induce significant electrophysiological changes in the brain through light stimulation.

Other authors have set out to investigate the effects on cognition of PBM and physical exercise. One example was carried out by Nagy and colleagues [63], where a placebocontrolled clinical trial was performed. Over 2 months, sixty older adults suffering from anemia and mild cognitive impairment were split into two groups to receive active or sham PBM, in addition to a moderate aerobic exercise. Their results showed that both groups significantly improved hemoglobin level and Montreal Cognitive Assessment Scale
(MoCa-B basic). Although the experimental group exhibited better results, it pointed out that while physical exercise positively impacts cognition, the effect of PBM alone should not be overlooked. Supplementary Table 1 provides a schematic overview of all the relevant experiments reported to allow for more direct comparability.

\section{Conclusions}

In conclusion, solid evidence around a substantial impact of near-infrared light on brain activity is building up, so future fNIRS studies should take into account the possible role played by light stimulation in their experimental designs. A careful investigation of whether the current devices and settings used for optical imaging can impact the participants cognitive performance would be dramatically important. Indeed, many of the findings from the fNIRS literature may hide the contribution of fNIRS per se, used as a measurement technique but neglected as a potential source of stimulation. Therefore, when assessing the efficacy of different interventions, such as physical exercise, on brain metabolism, researchers should include fNIRS as a factor and evaluate its possible effect. Failing to do so may hamper the capacity to estimate the true magnitude of intervention and lead to spurious results.

\section{Author contributions}

MM and NA designed the commentary. MM and NA wrote the manuscript. All authors contributed to editorial changes in the manuscript. All authors read and approved the final manuscript.

\section{Ethics approval and consent to participate} Not applicable.

\section{Acknowledgment}

Not applicable.

\section{Funding}

This work was supported by the Ministry of Science, Innovation and Universities (PSI2017-83893-R) and the Ministry of Economy and Business (PSI2017-90806-REDT).

\section{Conflict of interest}

The authors declare no conflict of interest.

\section{Supplementary material}

Supplementary material associated with this article can be found, in the online version, at https://www.imrpress.com/j ournal/JIN/20/4/10.31083/j.jin2004111.

\section{References}

[1] Jobsis F. Noninvasive, infrared monitoring of cerebral and myocardial oxygen sufficiency and circulatory parameters. Science. 1977; 198: 1264-1267.

[2] Mohammadi H, Gagnon C, Vincent T, Kassab A, Fraser S, Nigam A, et al. Longitudinal Impact of Physical Activity on Brain Pulsatil- 
ity Index and Cognition in Older Adults with Cardiovascular Risk Factors: A NIRS Study. Brain Sciences. 2021; 11: 730.

[3] Fabiani M, Low KA, Tan C, Zimmerman B, Fletcher MA, Schneider-Garces $\mathrm{N}$, et al. Taking the pulse of aging: Mapping pulse pressure and elasticity in cerebral arteries with optical methods. Psychophysiology. 2014; 51: 1072-1088.

[4] Tan CH, Low KA, Kong T, Fletcher MA, Zimmerman B, MacLin EL, et al. Mapping cerebral pulse pressure and arterial compliance over the adult lifespan with optical imaging. PLoS ONE. 2017; 12: e0171305.

[5] Fabiani M, Low KA, Tan C, Zimmerman B, Fletcher MA, Schneider-Garces N, et al. Taking the pulse of aging: Mapping pulse pressure and elasticity in cerebral arteries with optical methods. Psychophysiology. 2014; 51: 1072-1088.

[6] Wåhlin A, Ambarki K, Birgander R, Malm J, Eklund A. Intracranial pulsatility is associated with regional brain volume in elderly individuals. Neurobiology of Aging. 2014; 35: 365-372.

[7] Wood MD, Boyd JG, Wood N, Frank J, Girard TD, Ross-White A, et al. The Use of Near-Infrared Spectroscopy and/or Transcranial Doppler as Non-Invasive Markers of Cerebral Perfusion in Adult Sepsis Patients With Delirium: A Systematic Review. Journal of Intensive Care Medicine. 2021; 885066621997090.

[8] Robba C, Cardim D, Sekhon M, Budohoski K, Czosnyka M. Transcranial Doppler: a stethoscope for the brain-neurocritical care use. Journal of Neuroscience Research. 2019; 96: 720-730.

[9] Hoshi Y. Hemodynamic signals in fNIRS. Progress in Brain Research. 2017; 225: 153-179.

[10] Torricelli A, Contini D, Pifferi A, Caffini M, Re R, Zucchelli L, et al. Time domain functional NIRS imaging for human brain mapping. NeuroImage. 2014; 85: 28-50.

[11] Fantini S, Sassaroli A. Frequency-Domain Techniques for Cerebral and Functional Near-Infrared Spectroscopy. Frontiers in Neuroscience. 2020; 14: 300.

[12] Hernandez-Martin E, Gonzalez-Mora JL. Diffuse optical tomography in the human brain: a briefly review from the neurophysiology to its applications. Brain Science Advances. 2020; 6: 289-305.

[13] Izzetoglu M, Bunce SC, Izzetoglu K, Onaral B, Pourrezaei K. Functional brain imaging using near-infrared technology. Engineering in Medicine and Biology Magazine. 2007; 26: 38-46.

[14] Green MS, Sehgal S, Tariq R. Near-Infrared Spectroscopy: the New must have Tool in the Intensive Care Unit? Seminars in Cardiothoracic and Vascular Anesthesia. 2017; 20: 213-224.

[15] Hernandez-Martin E, Gonzalez-Mora JL. Diffuse Optical Tomography Using Bayesian Filtering in the Human Brain. Applied Sciences. 2020; 10: 3399.

[16] Habermehl C, Schmitz C, Koch SP, Mehnert J, Steinbrink J. Investigating hemodynamics in scalp and brain using high-resolution diffuse optical tomography in humans. Biomedical Optics and 3D Imaging. 2012.

[17] Haeussinger FB, Dresler T, Heinzel S, Schecklmann M, Fallgatter AJ, Ehlis A. Reconstructing functional near-infrared spectroscopy (fNIRS) signals impaired by extra-cranial confounds: an easy-touse filter method. NeuroImage. 2014; 95: 69-79.

[18] Agbangla NF, Audiffren M, Albinet CT. Use of near-infrared spectroscopy in the investigation of brain activation during cognitive aging: a systematic review of an emerging area of research. Ageing Research Reviews. 2018; 38: 52-66.

[19] Mendonca GV, Pezarat-Correia P, Vaz JR, Silva L, Almeida ID, Heffernan KS. Impact of Exercise Training on Physiological Measures of Physical Fitness in the Elderly. Current Aging Science. 2017; 9: 240-259.

[20] Hamilton GF, Rhodes JS. Exercise Regulation of Cognitive Function and Neuroplasticity in the Healthy and Diseased Brain. Progress in Molecular Biology and Translational Science. 2016; 135: 381-406.

[21] Kramer AF, Hahn S, Cohen NJ, Banich MT, McAuley E, Harrison CR, et al. Ageing, fitness and neurocognitive function. Nature. 1999; 400: 418-419.

[22] Strangman G, Boas DA, Sutton JP. Non-invasive neuroimaging using near-infrared light. Biological Psychiatry. 2002; 52: 679693.

[23] Haeussinger R, Bisconti S, Ulrich B. Motor Cortex Activity during Functional Motor Skills: an fNIRS Study. Brain Topography. 2016; 29: 42-55.

[24] Hernandez-Martin NT, Takakura H, Nishijo H, Ueda N, Ito S, Fujisaka M, et al. Cerebral Hemodynamic Responses to the Sensory Conflict Between Visual and Rotary Vestibular Stimuli: An Analysis With a Multichannel Near-Infrared Spectroscopy (NIRS) System. Frontiers in Human Neuroscience. 2020; 14: 125.

[25] Kirlilna E, Yu N, Jelzow A, Wabnitz H, Jacobs AM, Tachtsidis L. Identifying and quantifying main components of physiological noise in functional near infrared spectroscopy on the prefrontal cortex. Frontiers in Human Neuroscience. 2013; 7: 864.

[26] Masataka N, Perlovsky L, Hiraki K. Near-infrared spectroscopy (NIRS) in functional research of prefrontal cortex. Frontiers in Human Neuroscience. 2015; 9: 274.

[27] Hendrikx D, Smits A, Lavanga M, De Wel O, Thewissen L, Jansen $\mathrm{K}$, et al. Measurement of Neurovascular Coupling in Neonates. Frontiers in Physiology. 2020; 10: 65.

[28] Robbins TW. Dissociating executive functions of the prefrontal cortex. Philosophical Transactions of the Royal Society of London. Biological Sciences. 1997; 351: 1463-1461.

[29] Yuan P, Raz N. Prefrontal cortex and executive functions in healthy adults: a meta-analysis of structural neuroimaging studies. Neuroscience and Biobehavioral Reviews. 2015; 42: 180-192.

[30] Yanagisawa H, Dan I, Tsuzuki D, Kato M, Okamoto M, Kyutoku Y, et al. Acute moderate exercise elicits increased dorsolateral prefrontal activation and improves cognitive performance with Stroop test. NeuroImage. 2010; 50: 1702-1710.

[31] Lucas SJE, Ainslie PN, Murrell CJ, Thomas KN, Franz EA, Cotter JD. Effect of age on exercise-induced alterations in cognitive executive function: relationship to cerebral perfusion. Experimental Gerontology. 2012; 47: 541-551.

[32] Giles GE, Brunyé TT, Eddy MD, Mahoney CR, Gagnon SA, Taylor HA, et al. Acute exercise increases oxygenated and deoxygenated hemoglobin in the prefrontal cortex. NeuroReport. 2014; 25: $1320-1325$.

[33] Albinet CT, Mandrick K, Bernard PL, Perrey S, Blain H. Improved cerebral oxygenation response and executive performance as a function of cardiorespiratory fitness in older women: a fNIRS study. Frontiers in Aging Neuroscience. 2014; 6: 272.

[34] Dupuy O, Gauthier CJ, Fraser SA, Desjardins-Crèpeau L, Desjardins M, Mekary S, et al. Higher levels of cardiovascular fitness are associated with better executive function and prefrontal oxygenation in younger and older women. Frontiers in Human Neuroscience. 2015; 9: 66.

[35] Ichinose Y, Morishita S, Suzuki R, Endo G, Tsubaki A. Comparison of the Effects of Continuous and Intermittent Exercise on Cerebral Oxygenation and Cognitive Function. Advances in Experimental Medicine and Biology. 2020; 50: 209-214.

[36] Stone BL, Beneda-Bender M, McCollum DL, Sun J, Shelley JH, Ashley JD, et al. Understanding cognitive performance during exercise in Reserve Officers' Training Corps: establishing the executive function-exercise intensity relationship. Journal of Applied Physiology. 2020; 129: 846-854.

[37] Dietrich A, Audiffren M. The reticular-activating hypofrontality (RAH) model of acute exercise. Neuroscience and Biobehavioral Reviews. 2011; 35: 1305-1325.

[38] Mekari S, Fraser S, Bosquet L, Bonnéry C, Labelle V, Pouliot P, et $a l$. The relationship between exercise intensity, cerebral oxygenation and cognitive performance in young adults. European Journal of Applied Physiology. 2016; 115: 2189-2197.

[39] Ochi G, Yamada Y, Hyodo K, Suwabe K, Fukuie T, Byun K, et al. Neural basis for reduced executive performance with hypoxic exercise. NeuroImage. 2018; 171: 75-83.

[40] Pinti P, Tachtsidis I, Hamilton A, Hirsch J, Aichelburg C, Gilbert S, et al. The present and future use of functional near-infrared spectroscopy (fNIRS) for cognitive neuroscience. Annals of the New 
York Academy of Sciences. 2020; 1464: 5-29.

[41] Tachtsidis I, Scholkmann F. False positives and false negatives in functional near-infrared spectroscopy: issues, challenges, and the way forward. Neurophotonics. 2016; 3: 031405.

[42] Althobaiti M, Al-Naib I. Recent Developments in Instrumentation of Functional Near-Infrared Spectroscopy Systems. Applied Sciences. 2020; 10: 6522.

[43] Hamblin M, Huang YY. Photobiomodulation in the Brain. Elsevier: UK. 2019.

[44] Hamblin MR. Shining light on the head: Photobiomodulation for brain disorders. Biochimica et Biophysica Acta Clinical. 2019; 6: 113-124.

[45] Joensen J, Demmink JH, Johnson MI, Iversen VV, Lopes-Martins RÁB, Bjordal JM. The thermal effects of therapeutic lasers with 810 and $904 \mathrm{~nm}$ wavelengths on human skin. Photomedicine and Laser Surgery. 2011; 29: 145-153.

[46] Mochizuki-Oda N, Kataoka Y, Cui Y, Yamada H, Heya M, Awazu K. Effects of near-infra-red laser irradiation on adenosine triphosphate and adenosine diphosphate contents of rat brain tissue. Neuroscience Letters. 2002; 323: 207-210.

[47] Ilic S, Leichliter S, Streeter J, Oron A, DeTaboada L, Oron U. Effects of power densities, continuous and pulse frequencies, and number of sessions of low-level laser therapy on intact rat brain. Photomedicine and Laser Surgery. 2006; 24: 458-466.

[48] Zivin JA, Albers GW, Bornstein N, Chippendale T, Dahlof B, Devlin T, et al. Effectiveness and Safety of Transcranial Laser Therapy for Acute Ischemic Stroke. Stroke. 2009; 40: 1359-1364.

[49] McCarthy TJ, De Taboada L, Hildebrandt PK, Ziemer EL, Richieri SP, Streeter J. Long-term safety of single and multiple infrared transcranial laser treatments in Sprague-Dawley rats. Photomedicine and Laser Surgery. 2010; 28: 663-667.

[50] Johnstone DM, Moro C, Stone J, Benabid A, Mitrofanis J. Turning on Lights to Stop Neurodegeneration: the Potential of near Infrared Light Therapy in Alzheimer's and Parkinson's Disease. Frontiers in Neuroscience. 2016; 9: 500.

[51] Méndez M, Fidalgo C, Arias JL, Arias N. Methylene blue and photobiomodulation recover cognitive impairment in hepatic encephalopathy through different effects on cytochrome c-oxidase. Behavioural Brain Research. 2021; 403: 113164.

[52] Arias JL, Mendez M, Martínez J, Arias N. Differential effects of photobiomodulation interval schedules on brain cytochrome coxidase and proto-oncogene expression. Neurophotonics. 2020; 7 : 045011.
[53] Wang X, Tian F, Soni SS, Gonzalez-Lima F, Liu H. Interplay between up-regulation of cytochrome-c-oxidase and hemoglobin oxygenation induced by near-infrared laser. Scientific Reports. 2016; 6: 30540 .

[54] Farivar S, Malekshahabi T, Shiari R. Biological effects of low level laser therapy. Journal of Lasers in Medical Sciences. 2015; 5: 5862.

[55] Passarella S, Karu T. Absorption of monochromatic and narrow band radiation in the visible and near IR by both mitochondrial and non-mitochondrial photoacceptors results in photobiomodulation. Journal of Photochemistry and Photobiology Biology. 2014; 140: $344-358$.

[56] Hashmi JT, Huang Y, Osmani BZ, Sharma SK, Naeser MA, Hamblin MR. Role of Low Level Laser Therapy in Neurorehabilitation. Journal of Injury, Function, and Rehabilitation. 2010; 2: 292-305.

[57] Giordano J, Bikson M, Kappenman ES, Clark VP, Coslett $\mathrm{HB}$, Hamblin MR, et al. Mechanisms and Effects of Transcranial Direct Current Stimulation. Dose-Response. 2019; 15: 1559325816685467.

[58] Tanaka H, Haley AP. Beneficial neurocognitive effects of transcranial laser in older adults. Lasers in Medical Science. 2017; 32: 1153-1162.

[59] Kwater A, Gąsowski J, Wizner B, Kasprzyk Z, Cwynar M, Rewiuk $\mathrm{K}$, et al. Cardiovascular risk factors as determinants of cerebral blood flow - a cross-sectional and 6-year follow-up study. Blood Pressure. 2020; 29: 182-190.

[60] Tian F, Hase SN, Gonzalez-Lima F, Liu H. Transcranial laser stimulation improves human cerebral oxygenation. Lasers in Surgery and Medicine. 2017; 48: 343-349.

[61] Zomorrodi R, Loheswaran G, Pushparaj A, Lim L. Pulsed near Infrared Transcranial and Intranasal Photobiomodulation Significantly Modulates Neural Oscillations: a pilot exploratory study. Scientific Reports. 2019; 9: 6309.

[62] Lim L, Loheswaran G, Zomorrodi R, Saltmarche A, Chao L. Photobiomodulation: a novel approach to treating Alzheimer's disease. Photobiomodulation in the Brain. 2019; 56: 401-417.

[63] Nagy EN, Ali AY, Behiry ME, Naguib MM, Elsayed MM. Impact of combined photo-biomodulation and aerobic exercise on cognitive function and quality-of-life in elderly alzheimer patients with anemia: A randomized clinical trial. International Journal of General Medicine. 2021; 14: 141-152. 